\title{
The relevance of biotechnology in the development of functional foods for improved nutritional and health quality in developing countries
}

\author{
Lorraine L. Niba \\ Department of Human Nutrition, Foods and Exercise, 319 Wallace Hall, Virginia Polytechnic Institute and State \\ University, Blacksburg, VA 24061-0430, USA. Phone: 1-(540) 231-8763. E-mail: Iniba@vt.edu.
}

Accepted 21 November 2003

The quality of food and food plants can be modified and optimized to meet the nutritional and health needs of at-risk and compromised populations prevalent in most of the developing countries. High rates of malnutrition, infectious disease as well as diet-related diseases such as diabetes and hypertension are prevalent in many developing countries. These are as a result of compromised immune function, inadequate sources of nutritious and quality foods and limited access to healthy and suitable foods. Biotechnology and genetic modification techniques have been proposed and applied for the improvement of the quality of various food crops. These have typically been geared towards increasing yields and pest resistance of cash crops. There is considerably less emphasis however, toward improving quality with regard to fortification or functionality of foods and food plants. Functional foods have nutritional and physiological benefits and are applicable in disease prevention and management. The application of biotechnology techniques for the development of functional food plants with higher levels of bioactive components or increased availability of nutrients would greatly benefit most populations in developing countries and improve the health and nutritional status overall.

Key words: Biotechnology, functional foods, food quality, health, developing countries.

\section{INTRODUCTION}

High rates of malnutrition and escalating rates of dietrelated diseases such as diabetes and hypertension in developing countries are attributable to various concerns: inadequate sources of dietary protein, foods with high levels of anti-nutritional components and toxicants, a disproportionate amount of highly digestible, high- glycemic index carbohydrates which constitute the staple foods such as yams, maize and rice, and limited alternatives. In addition, socio-economic factors such as urbanization and migration to urban areas have led to changes in lifestyles to include imported highly processed foods and modification in eating patterns and food habits. 
In many rural areas, there are challenges of protein malnutrition as well as inadequate vitamin and mineral intake. Furthermore, these problems are compounded by inadequate prenatal nutrition which leads to deficiencies in both mothers and children. Nutritional needs of postpartum nursing mothers are often not met, subsequently leading to inadequate nutrition in children.

The quality of staple foods and other foods that are commonly consumed in most of these areas could be optimized to improve their quality.

While a recent survey of scientific experts around the world indicated that the top biotechnological need for developing countries was technology for diagnosis of infectious disease, other needs such as vaccines and increased nutrient content of food crops as well as combinatorial chemistry for drug discovery also ranked among the top ten needs (Daar et al., 2002). It is purported that without biotechnology, the yields and availability of food crops in developing countries, particularly in sub-Saharan Africa would be heavily compromised, leading to inadequate supplies by the year 2025 (Dyson, 1999; Thompson, 2002). Bouis et al. (2003) suggest that there are various ways in which biotechnology and genetic medication can be exploited for consumer benefit, particularly in developing countries: crop productivity, reduction in use of pesticides and improvement in micronutrient content of foods.

This review therefore seeks to describe the potential for modifying and optimizing quality of food crops to improve their functionality for improved health and nutritional status, as this would greatly contribute to disease prevention and management.

\section{BIOTECHNOLOGY, FOOD QUALITY AND FUNCTIONAL FOODS}

In certain areas, biotechnology and genetic modification techniques are being optimized for the production and development of healthy foods, and improvement in the levels and activity of biologically active components in food plants (phytochemicals). Biotechnology techniques in developing countries however have mostly been targeted at increasing yields of cash crops. Food crops or the improvement of food quality and functional foods have garnered much less attention.

Techniques applied in genetic modification include mutation breeding, improved conventional breeding, transgenic modifications, DNA insertion, gene transfer and somatic hybridization, (Bouis et al., 2003; Christou, 1997; Mazur, 2001; Mackay, 1991; Yan and Kerr, 2002).

While there has been some hesitation with regard to the acceptability and adoption of biotechnology products in certain developing countries, achievements such as the development of high-vitamin A rice have greatly increased the acceptability of biotechnology for human food applications among hitherto skeptical consumers. Furthermore, it provides insights into the potential for application of biotechnology in developing improved quality and functional foods for human nutrition and health, rather than simply for use in agricultural technology for improved yields and pest resistance. The production of increased levels of beta-carotene (the precursor to vitamin A) in plants is especially important, as its precursor, lycopene has been shown to have physiological chemo-preventive effects with regard to various cancers (Yan and Kerr, 2002). Furthermore, lycopene, commonly found in various carotenoid containing plants such as tomatoes and carrots, is an essential ingredient in maintaining eye health and vision.

Modifications that have been targeted and developed by various biotechnology companies include improvement in the oil content and composition of oil seeds such as legumes (Mazur, 2001; Mazur et al., 1999; Uzogara, 2000). Improvement in soybean oil quality includes stabilization of the unsaturated fatty acids by increasing levels of the antioxidant, vitamin $E$ (Yan and Kerr, 2002).

These successes indicate a relevant and important role for biotechnology in improving food quality and developing functional foods, particularly those targeted for needy populations in developing countries, such as children and pre-natal women.

\section{Starch Modification and Glycemic Index}

Genetic modification efforts geared at increasing the levels, yields of carbohydrates and dry matter of food plants have been largely successful. There is however, potential for modification and improvement of starch and carbohydrate quality.

Root and tuber crops such as cassava and yams are typically high in amylopectin with lower ratios of amylose to amylopectin. This yields highly digestible, highglycemic index foods. These high-glycemic index starches have been associated with diet-related conditions such as type 2 diabetes and insulin resistance.

Modification of starch to increase the amylose to amylopectin ratios would be greatly useful in improving glycemic index and physiological responses to the starchy foods that are prevalent in the diet in many developing countries.

Modifications in the starch synthesis pathway have been effected to modify ratios of amylose to amylopectin in potato and cassava starch (Visser et al., 1991, 1997). Improving the glycemic indices of staple starchy food crops such as cassava, yams, potatoes and grains will improve their nutritional quality, and subsequently have an effect on the prevalence of attendant disease conditions. 


\section{Bioactive Components}

Various commonly consumed food crops such as many vegetables, legumes and grains contain physiologically beneficial bioactive compounds such as anti-oxidant carotenoids, phenolics, alkaloids, glucosinolates and other phytochemicals.

Active compounds such as carotenoids from tomatoes, glucosinolates from Brassica vegetables, phytoestrogens from soybean and phenolics and antioxidants from various plants have been shown to protect and prevent against numerous diseases such as cancers and cardiovascular disease. These antioxidants are also associated with slowing the aging process and improving overall health. Phytoestrogens from Dioscorea species such as wild yam (Dioscorea villosa) have also been shown to possess numerous physiological benefits. Some sweet potato varieties have been shown to exhibit anti-diabetic potential.

Most of these bioactive components are plant secondary metabolites. Their levels and expression can be improved and optimized through genetic modification of their synthetic pathways. DellaPenna (1999) suggests that levels of these compounds could be increased in target plants by identifying candidate genes which can then be transferred by transgenic approaches to modify the synthetic pathways of interest.

Utilization of plants as 'bio-factories' for the production of vaccines in developing countries has been examined (DellaPenna, 1999; Thompson, 2002; Uzogara, 2000). This is attainable by use of transgenic plants for the production of adequate levels of desired vaccines and facilitates distribution to target populations who may otherwise not have access to such vaccines.

\section{Protein}

The daily protein requirement for optimal health status is estimated at about $0.8 \mathrm{~g} / \mathrm{kg}$ of body weight (FAO, 1985; Yan and Kerr, 2002). There is a need for substantial amounts of high-quality dietary protein. Unfortunately, in most developing countries, high quality proteins are scarce and of limited availability. Animal protein is particularly limited in areas of drought and in areas of poor soil quality or limited land. In urban areas, meats and animal products such as milk and eggs are mostly imported or even when produced locally, are generally expensive. As such there is a prevalence of protein malnutrition, particularly among dependent groups such as children and prenatal women who have the highest needs for high quality protein.

Genetic modification can be applied for improving the availability and quality of protein in food crops. This is primarily by modification of the amino acid composition and amounts, as has been done in some legumes and in soybean. Datta and Bouis (2000), have increased levels of lysine in soybean and canola by transgenic techniques. Application of hybrids to increase yields of beneficial crops from selected strains has also been examined (Mazur et al., 1999).

\section{MICRONUTRIENTS - BIOFORTIFICATION}

Depleted soils, rudimentary farming and agricultural techniques that characterize farming practices in poorer areas of most developing countries have led to food crops that are low in required micronutrients and vitamins. This in turn has led to high levels of micronutrient deficiencies and subsequently diseases associated with these deficiencies.

The effects of vitamin A, vitamin B12 and iron deficiencies are prevalent. There is a need therefore for better quality foods with adequate micronutrient levels. Commercial and industrial level fortification is used in most developed countries for foods and products in which processing has depleted nutrient levels. This is however expensive and not a feasible option in poorer countries.

Micronutrient content of products can be increased by insertion of genes that produce the desired nutrient. This is applicable for staples that are typically deficient. Introduction of soybean ferritin gene into rice results in increased iron content of rice (Goto et al., 1999). Ferritin being the primary storage protein, its gene coding sequence is inserted into rice plants, resulting in rice with up to three times the amount of iron (Goto et al., 1999). This has also been used in improving iron content of beans (Gura, 1999). This technique can possibly be examined for application in other grain cereals where iron absorption is compromised by the presence of phytates and other anti-nutritional components.

Genetic modification aimed at secondary compounds could also be beneficial in improving nutritional quality of foods. Iron absorption for instance is influenced by the presence of phytates and tannins in grains such as maize. Modified maize in which phytates have been reduced by insertion of the degrading enzyme (phytase) gene in the product, result in greater availability of iron, despite no change in overall content of iron (Bouis et al., 2003; Mendoza et al., 1998; Raboy, 1996). The increased iron absorption from low-phytic acid maize is particularly applicable in areas where maize and maize products are staples.

Other micronutrients with potential for application and improvement are zinc, iodine and potassium.

\section{BENEFICIAL FOOD CROPS}

Grain legumes contain high levels of macronutrients such as starch, protein and fiber. In addition, legumes such as 
Phaseolus beans and Vigna species, chick-pea and other such varieties contribute a significant proportion of the dietary energy in many tropical areas.

Legume consumption and utilization however is a distant second to grain cereals, roots and tuber crop consumption in most developing countries. Consumption of legumes is on the decline mainly due to lack of interest, limited research and development investment and lack of their exploitation as international cash crops. In addition, various legumes contain anti-nutritional factors such as cyanogenic glycosides and polyphenols. Furthermore, the long cooking times and the tedious cooking procedures required for preparing some legumes also decrease their utility and exploitation.

The macronutrients in legumes contribute to enhanced food quality. Legume proteins contain sulfur-containing amino acids that are typically deficient in grain cereals. Legume starches tend to be B-type starches that are less digestible, have lower glycemic index and result in lower corresponding increases in post-prandial blood glucose levels than most grain and root starches.

Modifications to improve legume quality would include increasing the yields, increasing protein content and augmenting levels of beneficial and desired amino acids, increasing starch content, reducing anti-nutritional and toxic factors such as cyanogenic glycosides, and development of easy-cooking varieties.

While genetic modification and bioengineering techniques have been applied to various crops, such techniques have only been marginally applied to legumes. However, various procedures have been suggested for modification of legumes. These include development of transgenic plants by use of Agrobacterium-mediated gene transfer and particle bombardment techniques (Christou, 1997). These techniques are proposed to be applicable in increasing levels of vitamins such as Vitamin A and B12 which are deficient in Phaseolus species (Christou, 1997). Additional applications include pest resistance in legumes, particularly aflatoxin in peanuts (Christou, 1997).

\section{FERMENTATION AND BIO-PROCESSING}

Fermented foods constitute an important part of the diet and food ingredients in many developing countries. These are primarily fermented grains and root crops, as well as curdled milks and milk products.

Fermented grain products are processed into staples such as gruels and dumplings, which serve as baby foods as well as for adult consumption. Fermented cassava and yam foods are staples in many parts of Africa. Fermentation is typically effected by bacteria and yeasts and facilitates preservation and storage. In addition, it serves as a means to reduce detrimental components such as cyanogenic glycosides. Levels of anti-nutritional factors such as haemagglutinins and tannins are depleted by fermentation (Lorri, 1993; Mbugua, 1992).

Fermentation markedly improves nutritional and health quality in food products. Protein content of certain grain foods is increased by fermentation. Furthermore, fermentation and staling results in modification of starches to form resistant starches which are associated with protection against colon cancer and gastrointestinal disease (Ahmed et al., 2000). In South Africa, for instance, a disparity in the rates of colon cancer between whites and black populations has been attributed to the consumption of fermented maize porridge which is high in complex and resistant starches (Ahmed et al., 2000).

Improvement and optimization of fermentation and bioprocessing in developing countries is applicable in improving quality and functionality of foods. This would simply be geared at improving already existing technologies as well as incorporating new technologies. It has been suggested that even though hitherto most fermentation processes used in developing countries do not use innocula or extrinsic cultures, these processes could be improved by using starter cultures, and also by backslopping, which entails application of brine from prior fermentation cycles (Holzapfel, 2002). These modifications could be effected by recombinant DNA technology, and have the advantage of being effective in decreasing the length of fermentation processes, and improving the overall quality and efficacy of fermentation (Holzapfel, 2002).

Controlled fermentation processes can be applied for the production of physiologically beneficial components, for increasing amounts of protein and for increasing the levels of complex carbohydrates and other fermentation products.

Protein content and quality in grain cereals is improved via fermentation as a result of the fact that trypsin inhibitors are depleted, increasing the digestibility of the proteins as well as availability of various amino acids (Holzapfel, 2002; Lorri, 1993; Mbugua, 1992)

Some fermentative bacteria such as Lactobacillus produce beneficial metabolic products which have antimicrobial properties. These include organic acids, hydrogen peroxide and various bacteriocins such as nicin (Holzapfel, 2002). This is an added benefit of optimized fermentation and bio-processing.

\section{PROBIOTIC MICRO-ORGANISMS}

Probiotic micro-organisms have great physiological import as their fermentation of complex, non-digestible carbohydrates in the colon produces short chain fatty acids that have numerous benefits. These short chain fatty acids are substrates for colonic cell metabolism and 
improve their resilience. In addition, the lowered $\mathrm{pH}$ of the colon acts to protect against proliferation of other bacteria. These short chain fatty acids are reported to play a role in prevention against colon cancer and other diseases. Most important, probiotic bacteria such as Bifidobacteria and various Lactobacilli improve immune status of the colon, and are involved in preventing and depleting pathogenic and infectious bacteria in the gastrointestinal tract such as Helicobacter pylori (Hamilton-Miller, 2003). Furthermore, probiotic bacteria are effective against lactose intolerance and diarrhea (Scheinbach, 1998).

Most commercial probiotics are produced by fermentation (Hansen, 2002). In addition, some strains of Bifidobacteria have been shown to be adherent to starches from maize, potato and oats (Crittenden et al., 2001). Improving cultivation and fermentation processes for the production of probiotic and beneficial bacteria is a viable avenue for dealing with the challenges of diseases such as diarrhea and the compromised status of gastrointestinal health in many developing countries.

\section{CONCLUSION}

Inadequate nutrition, sub-par quality foods and limited food processing capabilities have led to compromised, sub-par health status and a prevalence of diet-related diseases in many developing countries, most especially among children, prenatal and post-partum women. There is clear potential for the application of biotechnology and genetic modification as tools to combat these challenges and improve the situation of at-risk populations. While there is concerted and dedicated focus on combating more apparent challenges such as infectious disease and producing enough food to feed the growing populations, the quality of the foods produced ought to be an important consideration. The feasibility and cost of applying modern and emerging genetic modification technologies in these areas is certainly a daunting task, as sometimes the primary need is often just survival. However, as scientists and policy makers make progress towards alleviating these debilitating conditions, consideration should also be given to the possibility and potential for augmenting food quality and developing functional foods for improved nutritional and health status.

\section{REFERENCES}

Ahmed R, Segal I, Hassan H (2000). Fermentation of dietary starch in humans. Am. J. Gastroenterol. 95: 1017-1020.

Bouis HE, Chassy BM, Ochanda JO (2003). Genetically modified food crops and their contribution to human nutrition and food quality. Trends Food. Sci. Tech. 14:191-209.

Christou P (1997). Biotechnology applied to grain legumes. Field Crops Res. 53:83-97.
Crittenden R, Laitila A, Forssell $P$, Matto J, Saarela M, MattilaSandholm T, Myllarinen P (2001). Adhesion of bifidobacteria to granular starch and its implications in probiotic technologies. Appl. Environ. Microbiol. 67:3469-3475.

Daar AS, Thorsteinsdottir H, Martin DK, Smith AC, Nast S, Singer PA (2002). Top ten biotechnologies for improving health in developing countries. Nat. Genet. 32:229-232.

Datta S, Bouis HE (2000). Application of biotechnology to improving the nutritional quality of rice. Food Nutr. Bull. 21:451-456.

DellaPenna D (1999). Nutritional genomics: manipulating plant micronutrient to improve human health. Science 285:375-379.

Dyson T (1999). World food trends and prospects for 2025. Proc. Natl. Acad. Sci. 96:5929-5936.

FAO (1985). Food and Agricultural Organization, World Health Organization, United Nations University. Energy and protein requirement. Report of a Joint FAO/WHO/UNU Expert Consultation. World Health Organization Technical Report Series 724. Geneve, Switzerland: World health Organization.

Goto F, Yoshihara T, Shigemoto N, Toki S, Takaiwa F (1999). Iron fortification of rice seed by the soybean ferritin gene. Nat. Biotech. 17:282-286.

Gura T (1999). New genes boost rice nutrients. Science 285:994-995.

Hamilton-Miller JM (2003). The role of probiotics in the treatment and prevention of Helicobacter pylori infection. Int. J. Antimicrob. Agents 22:360-366.

Hansen EB (2002). Commerical bacterial starter cultures for fermented foods of the future. Int. J. Food Microbiol. 78:119-131.

Holzapfel WH (2002). Appropriate starter culture technologies for smallscale fermentation in developing countries. Int. J. Food Microbiol. 75:197-212.

Mackay GR (1991). Genetic Manipulation of the potato for improved processing. J Sci Food Agric. 57:449-458.

Mazur BJ (2001). Developing transgenic grains with improved oils, proteins and carbohydrates. Novartis Found Symp. 236: 233-239. discussion 240-1.

Mazur B, Krebbers E, Tingey S (1999). Gene discovery and product development for grain quality traits. Science 285:372-377.

Mendoza C, Viteri FE, Lonnerdal B, Young KA, Raboy V, Brown KH (1998). Effect of genetically modified, low-phytic maize on absorption of iron from tortillas. Am. J. Clin. Nutr. 68:1123-1127.

Mbugua SK, Ahrens RH, Kigutha HN, Subrahmanian V (1992). Effect of fermentation, malted flour treatment and drum drying on nutritional quality of uji. Ecol. Food Nutr. 28:271-277.

Raboy V (1996). Cereal low phytic mutants: a "global" approach to improving mineral nutritional quality. Micronutrients Agric. 2:15-16.

Scheinbach S (1998). Probiotics: Functionality and commercial status. Biotechnol Adv. 16:581-608.

Thompson JA (2002). Research needs to improve agricultural productivity and food quality, with emphasis on biotechnology. J. Nutr. 132:3441S - 3442S.

Uzogara SG (2000). The impact of genetic modification of human foods in the $21^{\text {st }}$ century: a review. Biotechnol. Adv. 18:179-206.

Visser R, Somhurst I, Kuipers G, Ruys N, Feenstra W, Jacobsen E (1991). Inhibition of the expression of granule-bound starch synthase in potato by anti-sense constructs. Mol. Gen. Genet. 225:289-296.

Visser R, Suurs L, Steeneken P, Jacobsen E (1997). Some physicochemical properties of amylose-free potato starch. Starch/Staerke 49: 443-448.

Yan L, Kerr PS (2002). Genetically engineered crops: their potential use for improvement of human nutrition. Nutr. Rev. 60:135-141. 